human nasal brushings and human and sheep lung slices. In the mouse nose and lung, the preferred configuration directed up to $\mathrm{x} 500$-fold higher transgene expression than the non-viral platform, for the lifetime of the animal. Transgene expression was observed in $14.1 \%$ of lung epithelial cells ( $p<0.0001$ compared to controls). Repeated monthly administration (3X) was possible without loss of expression or significant histological inflammatory reactivity. Reassuringly, insertion site profiling from transduced cell lines and mouse nose/lung samples reveals a pattern of integration comparable to those reported for other lentiviral vectors in clinical development, with no evidence for enrichment of insertion at undesirable loci. The stability of rSIV. F/HN vectors was evaluated in two bronchoscope catheters and two aerosol generation devices. Encouragingly for clinical translation, no significant loss of transduction activity was noted with any of these clinically relevant delivery devices $(\mathrm{p}=0.64)$. Delivery of rSIV. F/HN expressing CFTR to sheep lung resulted in CFTR mRNA at $\sim 30 \%$ the levels of endogenous ovine CFTR ( $p<$ 0.0001 compared to non-treated lobes), exceeding presumed therapeutic levels. With the majority of translational hurdles addressed, we are now entering toxicology studies and the final stages of pharmaceutical development prior to entering clinical trials.

\section{P204 IMMUNE RESPONSES TO SINGLE AND REPEATED ADMINISTRATION OF PGM169/GL67A: THE UK CF GENE THERAPY CONSORTIUM CLINICAL TRIALS}

${ }^{1} \mathrm{U}$ Griesenbach, ${ }^{1} \mathrm{AC}$ Boyd, ${ }^{2} \mathrm{R}$ Calcedo, ${ }^{3} \mathrm{~S}$ Cheng, ${ }^{1} \mathrm{~S}$ Cunningham, ${ }^{1} \mathrm{JC}$ Davies, ${ }^{1} \mathrm{M}$ Dewar, ${ }^{1}$ DR Gill, ${ }^{1} \mathrm{~A}$ Doherty, ${ }^{1} \mathrm{~T}$ Higgins, ${ }^{1} \mathrm{SC}$ Hyde, ${ }^{1} \mathrm{M}$ Manvell, ${ }^{1} \mathrm{C}$ Meng, ${ }^{1} \mathrm{JA}$ Innes, ${ }^{2} \mathrm{MP}$ Limberis, ${ }^{1} E$ Punch, ${ }^{3} \mathrm{R}$ Scheule, ${ }^{1} \mathrm{~N}$ Soussi, 'S Soussi, ${ }^{2} \mathrm{JM}$ Wilson, ${ }^{1}$ Ewfw Alton. ${ }^{1}$ UK Cystic Fibrosis Gene Therapy Consortium, London, Oxford, Edinburgh, UK; ' ${ }^{2}$ ene Therapy Program, Department of Pathology and Laboratory Medicine, University of Pennsylvania., Pennsylvania., USA; ${ }^{3}$ Genzyme-Sanofi, MA, USA

\subsection{6/thoraxjn-2014-206260.333}

Although most CF patients express CFTR protein (albeit mutant) and should therefore not recognise the wild-type CFTR protein as foreign, there is an inherent risk of activation of T-cells against the recombinant wild-type protein after gene therapy. In addition, we have previously shown that approximately 10\% of $\mathrm{CF}$ and non-CF subjects carry self-reactive CFTR-specific T-cells (Calcedo et al, Hum Gene Ther Clin Dev 2013). The reason for this is unknown and it is also unclear whether being positive for self-reactive T-cells affects disease severity or increases the risk of further T-cell activation after gene therapy.

As part of the UKCFGTC Phase I/IIa Pilot study [in which patients received a single dose $(5,10$ or $20 \mathrm{mls})$ of the non-viral formulation pGM169/GL67A] peripheral blood mononuclear cells (PBMC) were collected prior to dosing and approximately 4 weeks after nebulisation of $5 \mathrm{ml}(\mathrm{n}=2), 10 \mathrm{ml}(\mathrm{n}=6)$ or 20 $\mathrm{ml}(\mathrm{n}=17)$ of pGM169/GL67A. IFN-g ELISPOT to detect CFTR-specific T-cells in PBMC was performed. CFTR-specific T-cells were detectable in one patient pre- and post-dosing. In the remaining 18 patients we did not detect CFTR-specific Tcells. In addition we quantified anti-DNA antibodies (antinuclear and anti-cytoplasmic) in blood samples taken pre- and approximately 4 weeks post-dosing $(\mathrm{n}=7(5 \mathrm{ml}), \mathrm{n}=10$ $(10 \mathrm{ml})$ and $\mathrm{n}=17(20 \mathrm{ml})$. We did not observe any evidence for induction of anti-DNA antibodies after a single dose of pGM169/GL67A.

The UKCFGTC has now completed a Phase IIb multi-dose clinical trial in May 2014 (ClinicalTrials.gov identification number - NCT01621867). CF patients received 12 monthly doses of pGM169/GL67A (115 completed nine or more doses), or placebo by aerosol. PBMC were collected on two occasions prior to dose 1 to establish baseline levels for CFTR-specific Tcells, approximately 4 weeks after Dose 4 or 5 , and 2 to 4 weeks after Dose 12 and the ELISPOT was performed. In addition antiDNA antibodies were quantified. The Phase IIb trial will be unblinded in Summer 2014 to allow data analysis and all data will be presented at the conference.

Funded by the NIHR/EME Programme and the Cystic Fibrosis Trust.

\section{Lung function testing: new approaches}

\section{P205 MULTIPLE BREATH WASHOUTS IN CHILDREN CAN BE SIGNIFICANTLY SHORTENED WITHOUT COMPROMISING MEASUREMENT QUALITY}

${ }^{1} \mathrm{FA}$ Ahmad, ${ }^{2} \mathrm{SI}$ Inving, ${ }^{2} \mathrm{AB}$ Bush, ${ }^{2} \mathrm{LF}$ Fleming, ${ }^{2} \mathrm{SS}$ Saglani. ${ }^{1}$ Imperial College, London, UK; ${ }^{2}$ Royal Brompton and Harefield NHS Foundation Trust and Imperial College, London, UK

\subsection{6/thoraxjn-2014-206260.334}

Background Multiple-breath washout (MBW) is used to calculate a measure of ventilation heterogeneity, the lung clearance index (LCI), and requires tidal breathing until a previously inspired tracer gas concentration falls below 1/40th of the initial value, an arbitrary threshold. LCI is usually performed in triplicate, each taking 4-8 min to complete which may be taxing, particularly in young children and those with marked airflow obstruction. Shortened LCI is of interest since a reduction in the test time may increase feasibility and improve the clinical applicability of the measurement.

We hypothesised that LCI measurements could be reliably shortened. We also investigated whether shortened MBW was responsive to an intervention.

Patients and methods We calculated LCI from a fixed time point, and from a fixed number of breaths, as well as LCI and $25 \%$ (LCI0.25), 50\% (LCI0.5) and 75\% (LCI0.75) of 1/40th of the initial concentration of tracer gas (LCIstd) and the time saved, in children aged 6-16 years with asthma $(\mathrm{n}=21)$, cystic fibrosis $(\mathrm{CF}, \mathrm{n}=20)$ and primary ciliary dyskinesia (PCD, $\mathrm{n}=19)$, and healthy controls $(\mathrm{n}=17)$, aged $3-18$ years. Shortened LCI was also calculated in 29 asthmatic children pre and one month post one intra-muscular triamcinolone injection, part of our clinical severe asthma protocol.

Results Calculating shortened LCI from a fixed washout time or breath number was not reliable. However, all shortened LCI measurements from initial gas concentration correlated significantly with LCIstd in each disease group. LCI0.5 presented a balance between correlation with LCIstd (see figure) and timesaving. Mean proportion of time saved per washout, using LCI0.5, was 27\% (asthma), 28\% (CF) and 31\% (PCD). Furthermore, LCI0.5 was significantly reduced after triamcinolone in children with severe asthma (mean LCI0.5 pre, 5.5 and 5.1 post triamcinolone, $p=0.02$ ), and the change was similar to that demonstrated using LCIstd (mean LCIstd pre, 7.8 and post 7.0, $\mathrm{p}=0.001$ ).

Conclusion We show for the first time that LCI measurements can be shortened without loss of information in school-children with asthma, CF and PCD. LCI0.5 was the optimal surrogate measure for LCIstd when proportion of time saved, correlation 